\title{
Overexpression of elF-5A2 in mice causes accelerated organismal aging by increasing chromosome instability
}

\author{
Muhan Chen ${ }^{1}$, Jian-Dong Huang ${ }^{2}$, Hong Kui Deng ${ }^{3}$, Suisui Dong ${ }^{1}$, Wen Deng ${ }^{4}$, Sze Lan Tsang ${ }^{2}$, Michael SY Huen², \\ Leilei Chen', Tong Zan ${ }^{3}$, Gui-Xia Zhu ${ }^{2}$ and Xin-Yuan Guan ${ }^{1 *}$
}

\begin{abstract}
Background: Amplification of $3 q 26$ is one of the most frequent genetic alterations in many human malignancies. Recently, we isolated a novel oncogene elf-5A2 within the 3 q26 region. Functional study has demonstrated the oncogenic role of elF-5A2 in the initiation and progression of human cancers. In the present study, we aim to investigate the physiological and pathological effect of elF-5A2 in an elF-5A2 transgenic mouse model.

Methods: An elF-5A2 transgenic mouse model was generated using human elF-5A2 CDNA. The elF-5A2 transgenic mice were characterized by histological and immunohistochemistry analyses. The aging phenotypes were further characterized by wound healing, bone X-ray imaging and calcification analysis. Mouse embryo fibroblasts (MEF) were isolated to further investigate molecular mechanism of elF-5A2 in aging.

Results: Instead of resulting in spontaneous tumor formation, overexpression of elF-5A2 accelerated the aging process in adult transgenic mice. This included decreased growth rate and body weight, shortened life span, kyphosis, osteoporosis, delay of wound healing and ossification. Investigation of the correlation between cellular senescence and aging showed that cellular senescence is not required for the aging phenotypes in elF-5A2 mice. Interestingly, we found that activation of elF-5A2 repressed p19 level and therefore destabilized p53 in transgenic mouse embryo fibroblast (MEF) cells. This subsequently allowed for the accumulation of chromosomal instability, such as errors in cell dividing during metaphase and anaphase. Additionally, a significantly increase in number of aneuploidy cells $(p<0.05)$ resulted from an increase in the incidences of misaligned and lagging chromosomal materials, anaphase bridges, and micronuclei in the transgenic mice.
\end{abstract}

Conclusion: These observations suggest that elf-5A2 mouse models could accelerate organismal aging by increasing chromosome instability.

Keywords: elF-5A2 aging, chromosome instability, transgenic mouse, oncogene

\section{Background}

It is believed that the process of malignant tumor is a multiple-step process caused by the accumulation of abnormal expression of oncogenes and tumor suppressor genes. Therefore, the identification of commonly amplified chromosomal region and corresponding overexpressed oncogenes within the region is imperative to understand the molecular mechanism of cancer

\footnotetext{
* Correspondence: xyguan@hkucc.hku.hk

'Department of Clinical Oncology, Faculty of Medicine, The University of

Hong Kong, 21 Sassoon Road, Hong Kong, China

Full list of author information is available at the end of the article
}

development. Amplification of chromosomal region $3 q 26$ is frequently detected in solid tumors, including ovarian [1], lung [2], esophageal [3], prostate [4], breast [5], and nasopharyngeal cancers [6], suggesting that $3 q 26$ contains an oncogene(s) related to the pathogenesis of human cancers. Using hybrid selection approach, we identified a candidate oncogene, eukaryotic translation initiation factor 5A2 (eIF5-A2), from 3q26.2 [7,8]. The functions of eIF-5A2 are mainly revealed in cancer initiation and progression. The tumorigenic ability of eIF5-A2 has been demonstrated by several in vitro evidences: eIF-5A2 stably transfected LO2 cells

\section{Biomed Central}


(immortalized human liver cell line) displayed increased colony formation in soft agar and xenograph formation in nude mice; reduction of eIF-5A 2 in ovarian cancer cell line UACC-1598 inhibits cell growth; and the oncogenic ability of eIF5-A2 can be blocked by eIF-5A2 silencing [7-9]. Previously, we showed that overexpression of eIF-5A2 at the protein level was significantly associated with the advanced stages of ovarian cancer [8]. Similarly, Marchet et al. recently reported that overexpression of eIF-5A2 is associated with a higher risk of lymph node metastasis in human gastric adenocarcinomas [10]. Recently, Zender et al. identified that eIF-5A2 is amplified in human cancer using representational oligonucleotide microarray analysis (ROMA), and is required for proliferation of XPO4-deficient tumor cells and promotes hepatocellular carcinoma in mice [11]. However, the in vivo function of $e I F-5 A 2$ is still not clear.

To further investigate the functions of the $e I F-5 A 2$ gene per se, we generated eIF-5A2 transgenic mice. Unexpectedly, the $e I F-5 A 2$ transgenic mice exhibit accelerated organismal aging phenotypes instead of forming spontaneous tumors. Cancer is assumed to be a disease of accumulated aged cells because of the close link between incidence of the cancer and the aging process. For decades, age has been regarded as the largest risk factor associated with the cancer initiation, which is supported by cancer incidence rising exponentially with age $[12,13]$. Mechanistically, genomic instability in somatic cells has been implicated as one of the major stochastic causes of aging [14-16]. Furthermore, cellular senescence, a potential in vitro counterpart of organismal aging, was demonstrated by several recent studies as a barrier to tumorigenesis, and contributes to the cytotoxicity of certain anticancer agents [17]. However, it is not clear whether aging functions the same way as cellular senescence to suppress tumor initiation in vivo. Also, there is no direct evidence to elucidate the functional contribution of senescent cells towards the onset of aging.

In this study, we found that eIF-5A2 overexpression triggers premature aging in multiple organs of the transgenic mice. We further demonstrated that supraphysiological expression of eIF-5A2 repressed p19 expression and therefore impaired p53 levels. This allowed for the accumulation of chromosomal instability, which ultimately led to organismal aging. To our knowledge, this study revealed for the first time a role of putative oncogenic eIF-5A2 in accelerating the aging process. Moreover, the accelerated aging process shows tumor suppression effects in vivo, and cellular senescence is not required for this single genetic change caused aging.

\section{Methods}

\section{Generation of elF-5A2 transgenic mice}

A 462 bp human eIF-5A2 cDNA fragment was cloned into the pCAGGS vector. The linearized constructs were injected into one-cell-stage F1 mouse embryos, which were transplanted into pseudo-pregnant females. All resulting pups were screened for the presence of the transgene using a pair of primers from the vector sequence and a pair of primers from the human eIF-5A2 gene. For studies relying on timed pregnancies, mating pairs were established and mice were monitored daily for vaginal plugs, the presence of which would indicate 0.5 days post-copulation. Animal experimentation was done in accordance with the guidelines of the University of Hong Kong regarding the care and use of laboratory animals.

\section{Western and Northern blot analysis}

For Western blot analysis, Protein lysates were prepared with RIPA buffer $(1 \times$ PBS, $1 \%$ Nonidet P40, $0.5 \%$ sodium deoxycholate, $0.1 \%$ SDS and protease inhibitor cocktail). About $10 \mu \mathrm{g}$ of lysate was separated by SDS-polyacrylamide gel electrophoresis, transferred to a PVDF HybondP membrane (Amersham Pharmacia Biotechnology, Piscataway, NJ), and detected by antibodies for eIF-5A2 (a mouse monoclonal antibody raised against the 54 residues of eIF-5A2), p53 (Zymed, San Francisco, CA), p21 (Upstate, Temecula, CA), p19 (Upstate, Lake Placid, NY), CDK4 (Cell Signaling Technology, Beverley, MA), and $\gamma$ tubulin (Sigma, St. Louis, MO). For Northern blot analysis, total cellular RNA was prepared using the TRIzol/ chloroform method. Twenty microgram of RNA was size fractionated, transferred to a nylon membrane, and hybridized with a ${ }^{32} \mathrm{P}$-labeled human $e I F-5 A 2$ specific probe as described previously $[7,8]$.

\section{Histological analysis, immunohistochemistry, senescence and BrdU incorporation assay}

Fresh mouse tissues were fixed in $4 \%$ cold paraformaldehyde in PBS, processed into serial paraffin sections, and stained with Mayer's hematoxylin-eosin staining. Immunohistochemistry (IHC) was performed using the following antibodies: eIF-5A2 (1:500 dilution), PCNA (1:500 dilution, Santa Cruz Biotechnology, Santa Cruz, CA). Internal standardization was achieved by comparing only images stained with the same antibodies in the same experiment, captured with identical parameters, and scaled and displayed identically. For $\beta$-galactosidase staining, MEF cells were stained for senescence-associated acidic $\beta$-galactosidase activity according to the manufacturer's protocol (Cell Signaling Technology, Beverley, MA). BrdU (100 mg/g of body weight) was injected i.p. into pregnant females. Then the animals were killed $2 \mathrm{~h}$ after injection and the mouse tissues or embryos were fixed in $4 \%$ paraformaldehyde at $4{ }^{\circ} \mathrm{C}$ overnight. Then the sections were processed for stained with BrdU staining kit (ZYMED) according to the manufacturer's protocol. 


\section{Wound healing experiments}

4-month old mice were anaesthetized with methoxyfluorane, and the dorsum was shaved and cleaned with alcohol. Three equidistant 1 -cm full-thickness incisional wounds were made through the skin and panniculus carnosus muscle. Wounds were measured at days 1, 2, 3 and 4 post-wounding, and wounded skin specimens were collected and bisected for histology at day 4 postwounding.

\section{Bone X-ray imaging and calcification analysis}

Individual mice were subjected to an X-ray imager for the detection of kyphosis and osteoporosis. In brief, mice were anaesthetized with $2 \%$ isofluorane and images were taken by a 600P X-ray mammogram machine (General Electric Co., Albuquerque, NM) with a dose of $15 \mathrm{kV}$ for $100 \mathrm{sec}$. For calcification analysis, embryos were eviscerated and the skin was removed. The embryos were fixed in 95\% ethanol and stained in Alcian blue solution and Alizarin red solution overnight as described previously [18].

\section{Isolation of mouse embryo fibroblast (MEF) cells}

E13.5 embryos were digested at $37^{\circ} \mathrm{C}$ for $10 \mathrm{~min}$ in $0.2 \%$ trypsin (Sigma, St. Louis, MO) in PBS (pH 7.4). The cell suspension was cultured in Dulbecco's modified Eagle's medium (DMEM) supplemented with $10 \% \mathrm{FBS}$ at $37^{\circ} \mathrm{C}$. For cell proliferation analysis, $5 \times 10^{4}$ wild-type and eIF$5 A 2$ transgenic MEF cells were plated on 6-well plates and cell numbers were counted every day for up to 6 days. For flowcytometry test, cells were fixed in $70 \%$ ethanol, stained with propidium iodide, and analyzed by flow cytometer.

\section{Cytogenetic analysis, FISH and SKY}

Metaphase spreads were prepared from either cultured MEF cells or bone marrow lymphocytes. Metaphases were harvested from cultured MEFs by Colcemid treatment $(0.03 \mu \mathrm{g} / \mathrm{ml})$ for $3 \mathrm{hr}$ or from bone marrow lymphocytes in tested mice by colchincine treatment $(3 \mathrm{mg} / \mathrm{kg}$, intraperitoneal injection). Metaphase spreads were stained by standard trypsin-Giemsa banding method and analyzed under microscope as described previously [7].

For fluorescence in situ hybridization (FISH) analysis, the whole transgene construct was used as a probe, which was labeled with Spectrum Red-dUTP by nick translation (Life Technologies, Inc.). The labeled probe was then hybridized to prebanded wild-type or eIF-5A2 transgenic MEF metaphase chromosomes as described previously [7].

Spectral karyotyping (SKY) was performed using a SKY probe (Applied Spectral Imaging, Migdal Ha'Emek, Israel) as described previously $[19,20]$. The signal detection followed the recommendations of the SKY probe manufacturer. SKY image capturing and karyotyping were performed using the SkyVision Imaging System equipped with a Zeiss Axioplan 2 fluorescence microscope.

\section{Telomerase activity assay}

The telomere repeat amplification protocol (TRAP) assay was performed using the TRAPEZE kit (Invitrogen, New York, NY) following the manufacturer's protocol. Except for negative control (lysis buffer), $0.05 \mu \mathrm{g}$ protein was used for each PCR, which was run for 33 cycles.

\section{Statistical analysis}

Statistical analysis was performed using the SPSS software (SPSS Standard version 8.0). Significance of difference was analyzed using Student's t tests. A significant difference was considered when the $p$ value was less than 0.05 .

\section{Results}

\section{Generation of elF-5A2 transgenic mouse lines}

To characterize the role of eIF-5A2 in vivo, we generated transgenic mouse lines overexpressing human $e I F-$ $5 A 2$ ubiquitously. Pronuclear injection of the pCAGGSeIF-5A2 construct resulted in the generation of three founder mice, as identified by genomic Southern blot analysis and PCR (Figure 1A). To confirm the single integration event, an eIF-5A2 probe was used to hybridize to the metaphase spreads from bone marrow lymphocytes by fluorescence in situ hybridization (FISH). The transgene was mapped to one chromosome site in line 11 (Figure 1B). All the founders were able to transmit the transgene to their offspring in the expected Mendelian ratio. Three lines were expanded, and the expression of the transgene was confirmed in two out of three founder lines (10 and 11). Expression of eIF-5A2 at the RNA level was detected by RT-PCR and Northern blot hybridization using liver and testis tissues (Figure 1C). Also, EIF-5A2 protein expression was demonstrated by immunohistochemistry (IHC) and Western blot analysis (Figure 1D) in multiple organs. Of note, no difference was observed in the expression of murine endogenous eIF-5A2 among the founders and wild-type controls. To ensure that the observed phenotypes were not a result of genomic insertion positional effects, we performed all experiments with both transgenic lines and obtained similar results for both lines. Expression of transgene eIF-5A2 in trnasgenic mice could be detected in all tested tissues by RT-PCR (Figure 1E).

\section{Characterization of elF5-A2 transgenic mice}

To determine the effects of eIF-5A2 on mouse development, protein expression in13.5-day embryos and 12week old mice was studied. On embryonic day 13.5, expression of eIF-5A2 was detected in the developing 


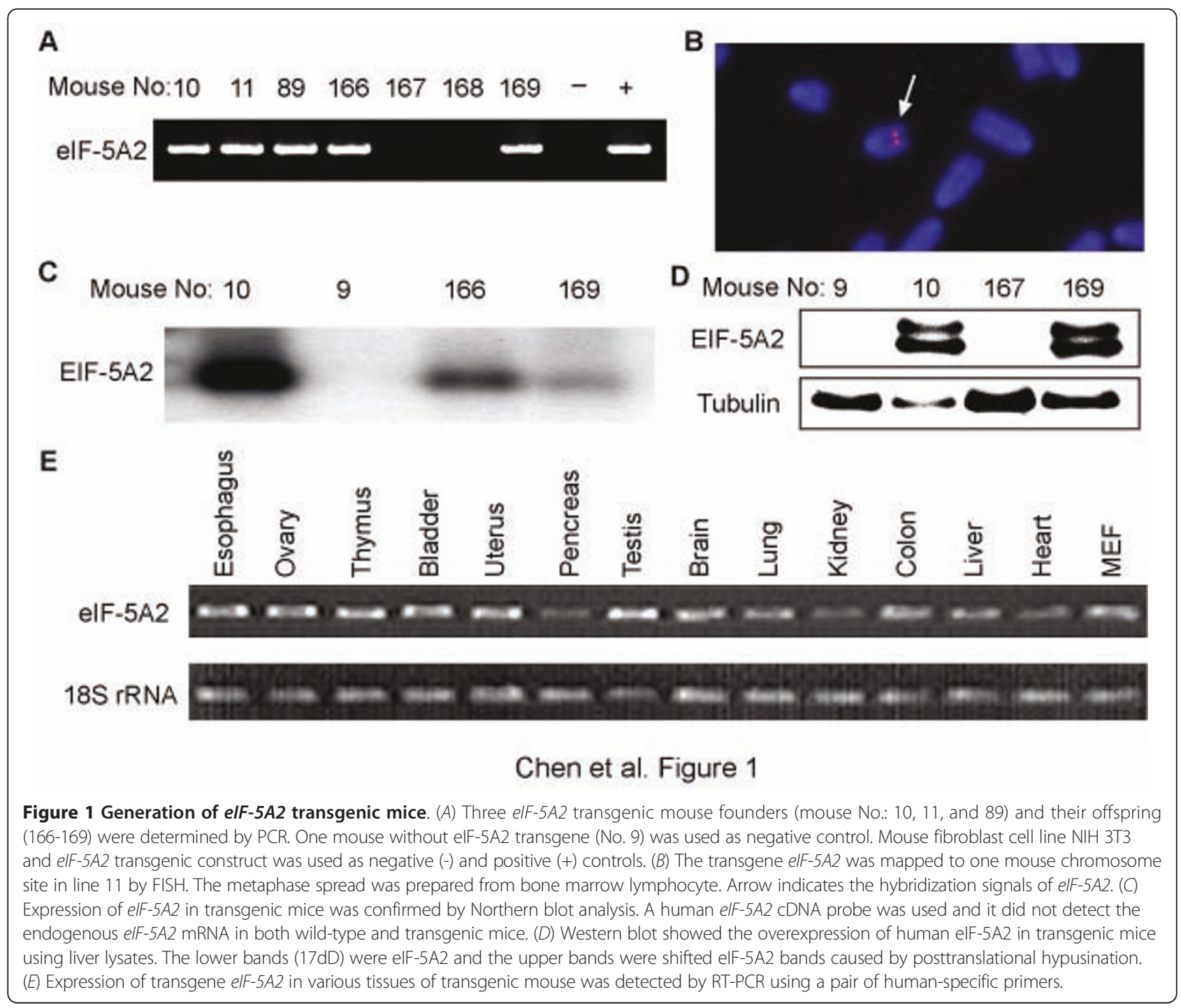

brain, liver and cartilage (Figure 2). At 12-week of age, EIF-5A2 protein expression was detected in all the tested adult tissues, including brain, lung, heart, liver, kidney, spleen, testis, uterus and ovary (sure 3A). Histological analysis showed that the morphologies of all tested tissues in eIF-5A2 transgenic embryos/mice were indistinguishable from their wild-type counterparts. To test the effects of eIF-5A2 overexpression on cell proliferation, BrdU incorporation assay and proliferating cell nuclear antigen (PCNA) immunostaining was applied to measure the proportion of cells in $\mathrm{S}$ phase in E13.5 embryos and12-week-old mice, respectively. However, the frequencies of BrdU positive cells in transgenic and wild-type embryos (56.3 \pm 7.81 vs. $52.73 \pm 9.14$, respectively; $p>0.05)$ and PCNA positive cell percentages in transgenic and wild-type mice $(46.2 \pm 10.98$ vs. $37.87 \pm$ 10.44, respectively; $p>0.05$ ) were comparable (Figure 2 and $3 \mathrm{~A})$.
No spontaneous tumor formation was observed in $e I F-$ $5 A 2$ transgenic mice $(n=452)$ during a period of 3 years. Alcohol intoxication, an efficient way to induce liver tumorigenesis in cancer-prone rodents [21], was used to induce liver lesions in 12-week-old eIF-5A2 transgenic mice $(n=20)$ and wild-type littermates $(n=12)$. After 13 weeks of alcohol treatment, livers of transgenic and wildtype mice were harvested and examined by histologists. No visible liver tumors or precancerous lesions were detected in all of the tested animals (Figure 3B). This suggests that eIF-5A2 overexpression alone does not increase the susceptibility of cancer onset in mice.

\section{Exhibition of accelerated aging phenotypes in elF-5A2 transgenic mice}

eIF-5A2 transgenic mice were indistinguishable from their wild-type siblings at birth. However, phenotypes of transgenic mice and wild-type littermates could be 


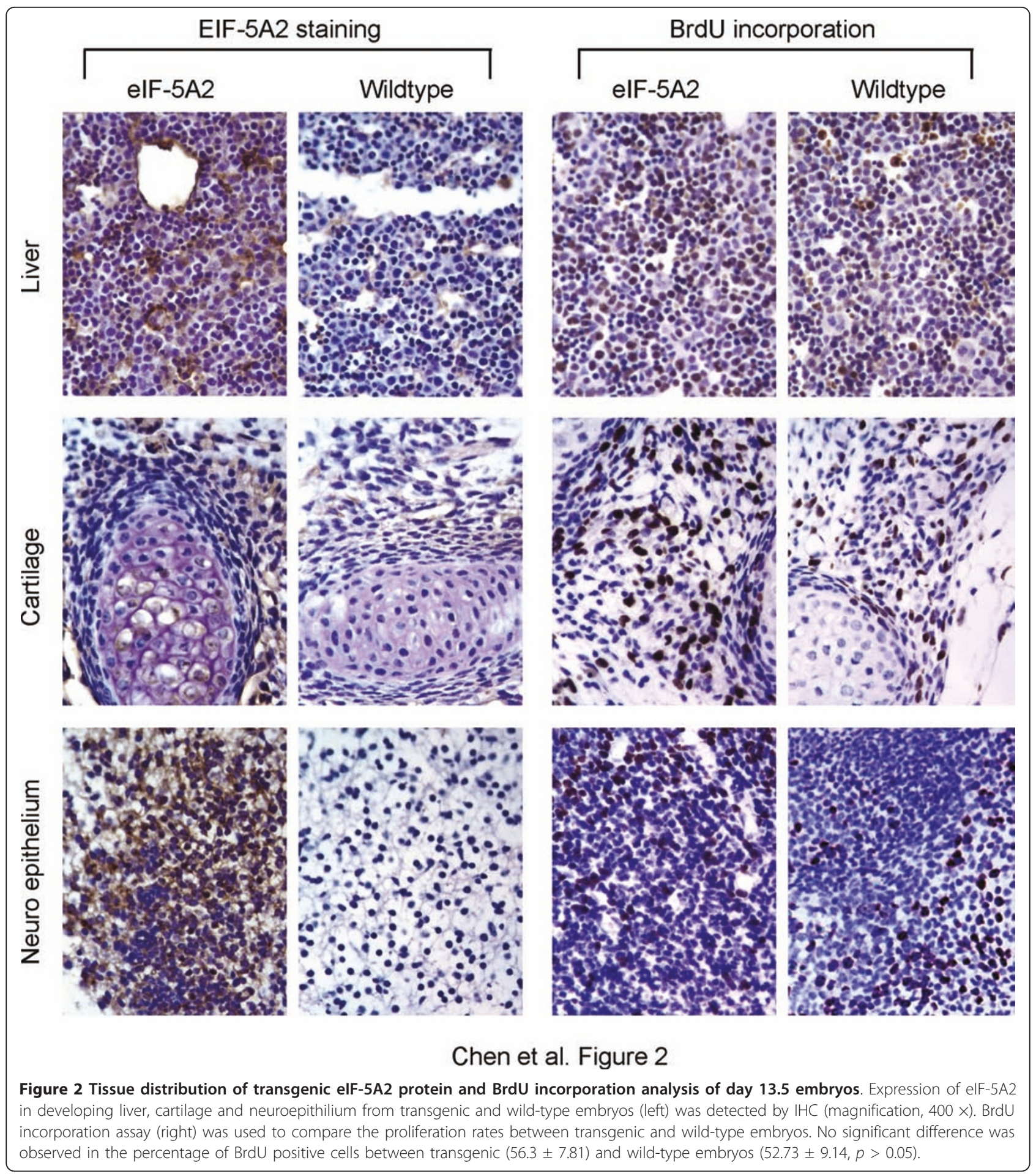

distinguished from postnatal week 3 according to body size and weight. From 3 weeks of age, the growth rate of transgenic mice was significantly reduced (about 20$40 \%, P<0.05$, Student's $t$ tests) compared with wildtype controls (Figure 4A). At 5 months of age, the body weights of transgenic male mice $(n=11)$ and wild-type male littermates $(\mathrm{n}=8)$ were compared, and we found the mean body weight of wild-type mice $(57 \pm 3.08 \mathrm{~g})$ was significantly higher than that of transgenic littermates (37.1 $\pm 4.83 \mathrm{~g}, p<0.05$, Student's $t$ tests) (Figure 4B). Strikingly, most of the transgenic mice died at 7-9 months without any apparent causes of death or visible 


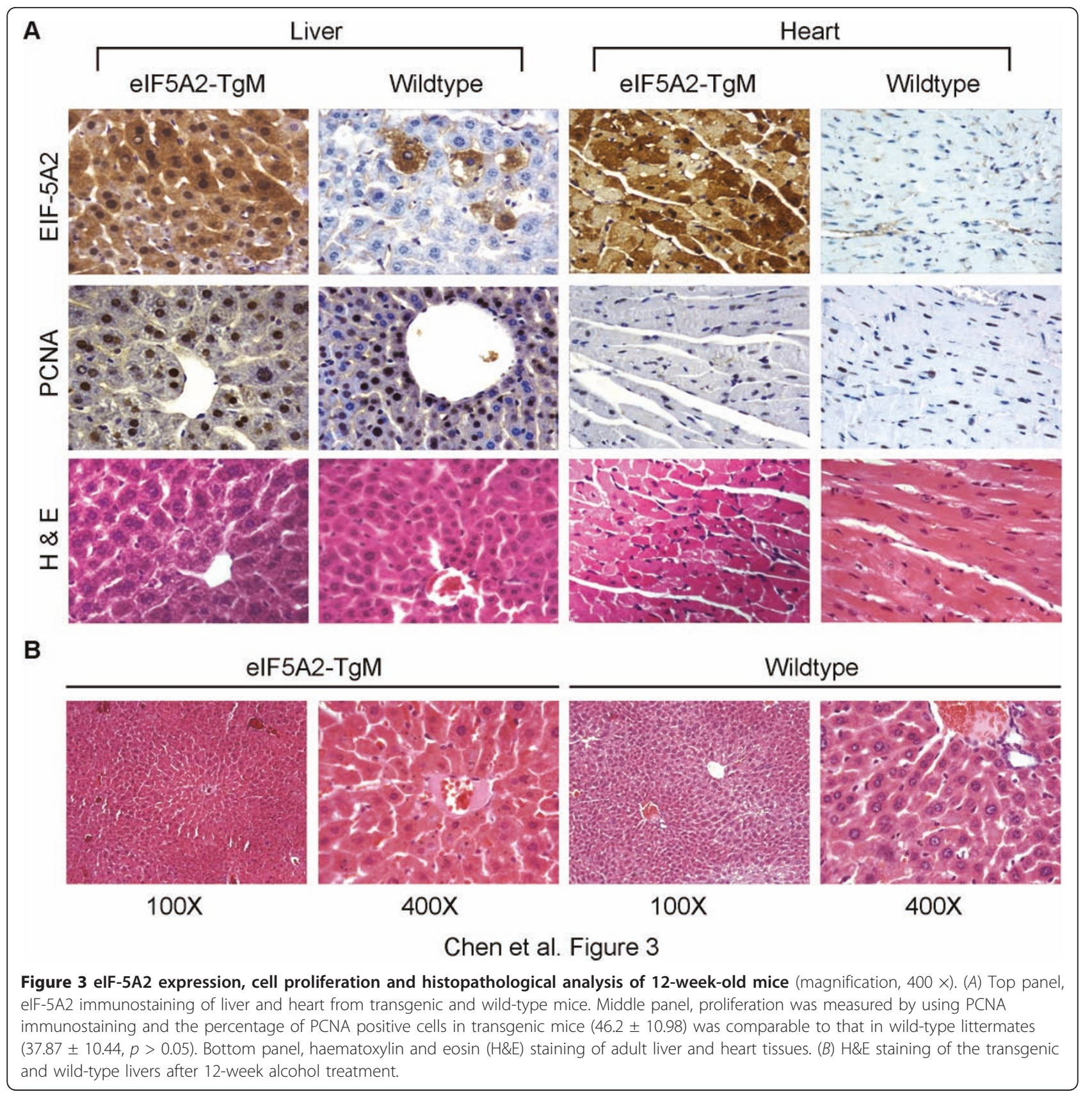

changes observed by autopsy. The average lifespan of eIF-5A2 mice is 8 months (Figure $4 \mathrm{C}$ ).

\section{Skin aging phenotypes in elF-5A2 mice}

Reduction of the capacity to respond to stresses such as wound healing is often associated with human aging [22]. Wound healing ability was compared between eIF$5 A 2$ transgenic mice and their wild-type siblings. We found that wound healing was significantly delayed $(p<$ 0.05 , Student's $t$ tests) in transgenic mice compared with their wild-type littermates (Figure 4D). Histological analysis showed that the ability of re-epithelialization in the edges of the wound was remarkably reduced in transgenic mice compared to their wild-type controls (Figure 4E). In addition, chronic skin lesions frequently occurred in eIF-5A2 transgenic mice due to the unhealing wounds or scratches (Figure 4F).

\section{Skeletal degeneration in elF-5A2 transgenic mice}

Radiographs were taken to examine for potential skeletal degeneration in eIF-5A2 transgenic mice $(n=6)$ and their wild-type siblings $(n=5)$ at 24 weeks of age. Almost 

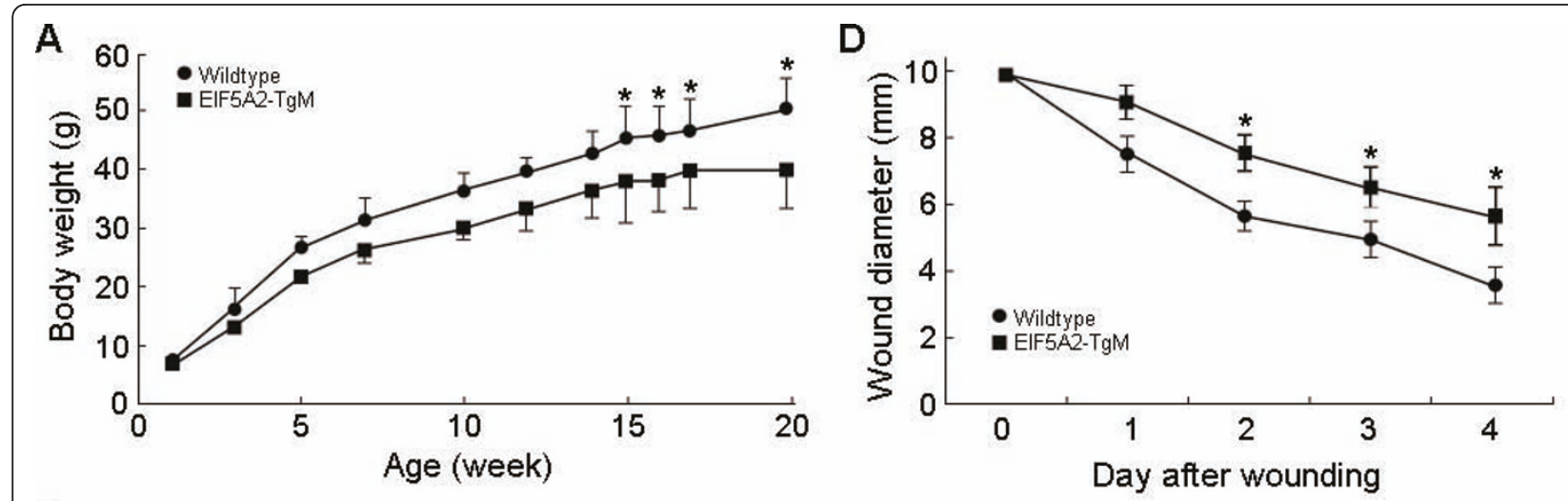

B
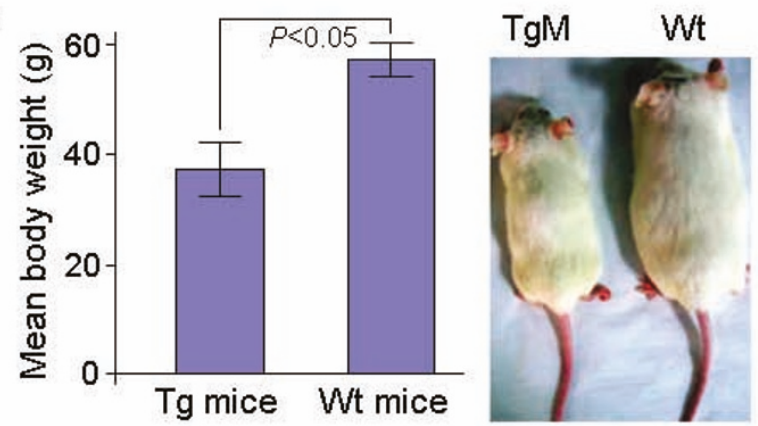

E

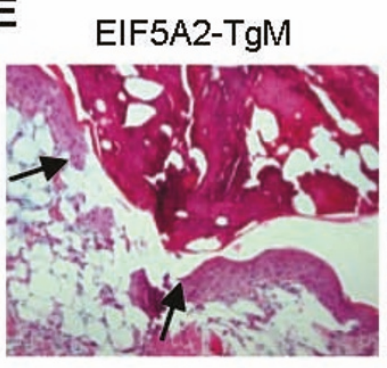

Wildtype mouse

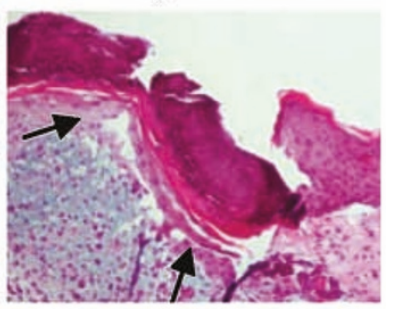

C
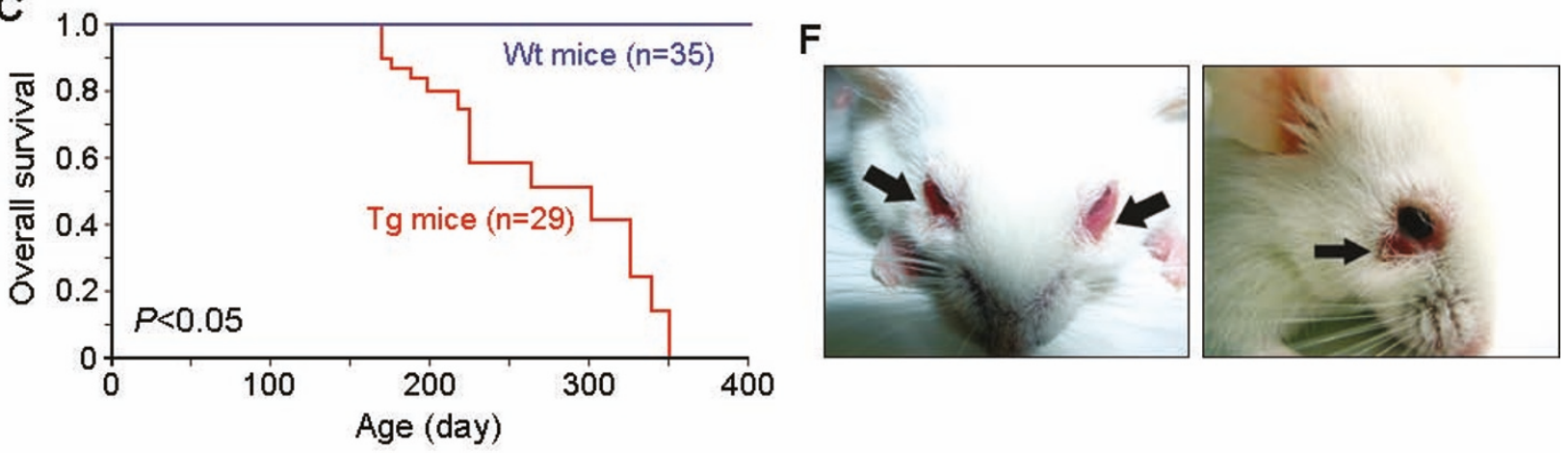

Chen et al. Figure 4

Figure 4 Aging-related phenotypes in elF5A2 transgenic mice. (A) Cumulative plot of body weight versus age of male elF-5A2 transgenic mice $(n=11)$ and their wild-type siblings $(n=8) .{ }^{*} P<0.05$. (B) Quantification of the mean body weight of 5-month-old male elf-5A2 transgenic mice and wild-type littermates is shown in the left $(p<0.05)$, and the representative image of a 24-week-old transgenic mouse and wild-type sibling was shown in the right. The body size of the transgenic mouse was significantly smaller than its wild-type sibling $(P<0.05)$. $(C)$ Kaplan-Meier survival curve of male transgenic mice $(n=29$, red line) and wild-type controls $(n=35$, blue line). (D) Comparison of wound healing rates between 4-month-old elF5A2 transgenic mice (15 wounds in 5 transgenic mice) and their wild-type siblings (12 wounds in 4 mice). * $P<0.05$. (E) Representative HE-stained section at day 4 post-wounding in an elF-5A2 transgenic mouse (left) and its wild-type sibling (right). The ability of re-epithelialization in the edges of wound was remarkably reduced in the transgenic mouse compared with its wild-type control (indicated by arrows). (F) Chronic skin lesions in elF-5A2 transgenic mice (indicated by arrows).

all the eIF-5A2 transgenic mice showed varying severity of kyphosis at 24-week of age (Figure 5A). In addition, the transgenic mice exhibited severe osteoporosis by radiographic analysis compared with their wild-type siblings (Figure 5B). We also stained the whole mouse skeletons with Alcian blue and Alizarin red to look at bone and cartilage. Interestingly, we found that 2-week-old transgenic mice showed several signs of delay of ossification. Compared to their wild-type siblings, transgenic pups had wide fontanelle and cranial sutures (Figure 5C and 5D). In the hind limb, clear ossification of the patella at the knee joint was observed in wild-type mice, but not in transgenic mice (Figure 5E). These data suggest that eIF-5A2 overexpression impairs ossification during 


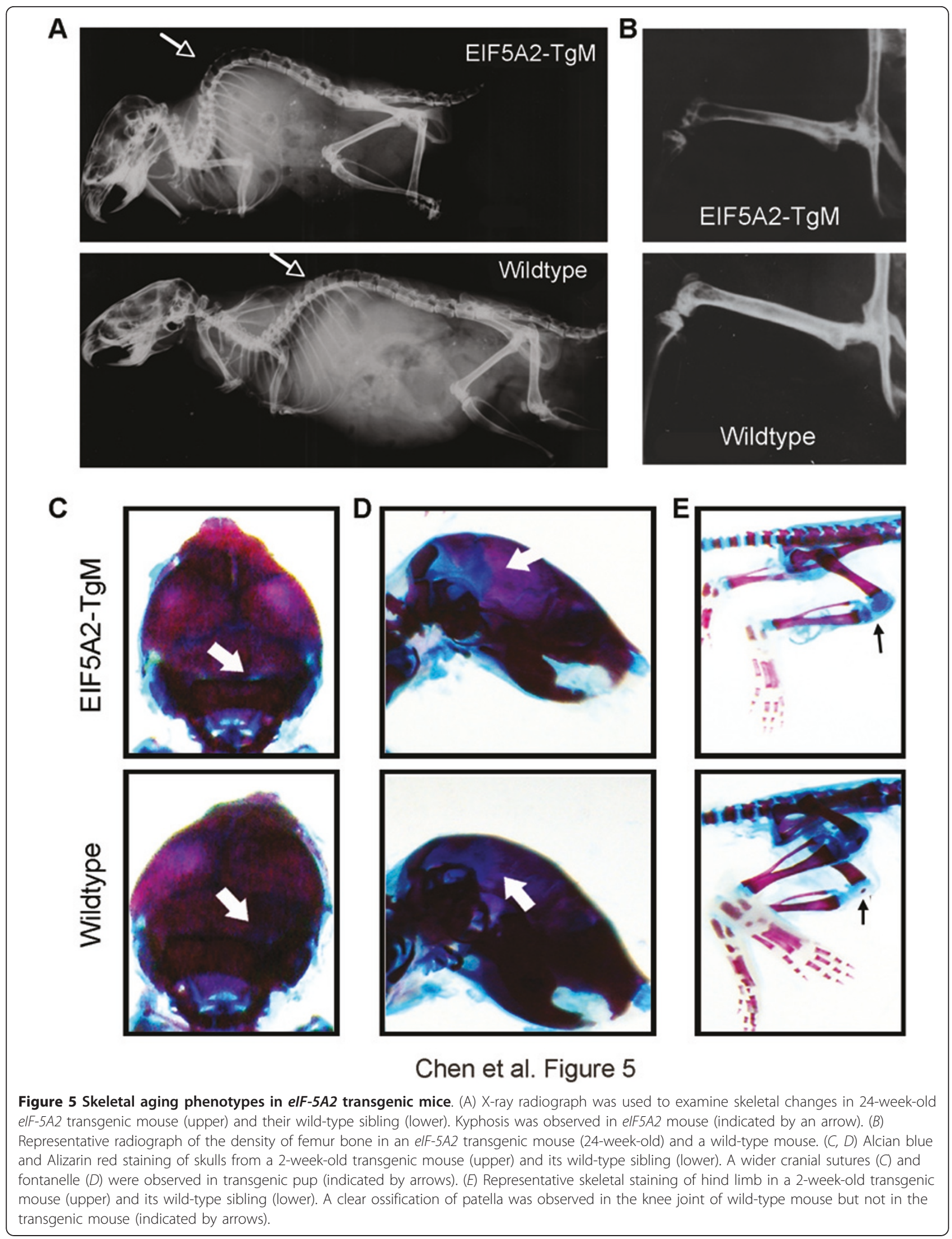


development and contributes to osteoporosis and spine degeneration in adulthood.

\section{elF-5A2 activation is insufficient for triggering cellular senescence}

Cellular senescence can be triggered by oncogene activation and agents that damage DNA or alter chromatin structure [13,17]. Given our observation of organismal aging in eIF-5A2 mice, we further investigated whether cellular senescence exists under these circumstances. Therefore, MEFs from eIF-5A2 transgenic mice and their wild-type littermates were characterized. eIF-5A2 MEFs were morphologically indistinguishable from the wildtype counterparts at both early and late passages. The activity of senescence associated $\beta$-galactosidase (SA- $\beta$ gal), a cellular index of senescence, was eximined and no difference was found between $e I F-5 A 2$ transgenic and wild-type MEFs (Figure 6A). We also stained the cryostat sections of skin, testis and liver, and did not detect any SA- $\beta$-gal positive cells in vivo (not shown).

The p53 tumor suppressor pathway in senescence has been well studied and serves as a critical player that mediates both replicative and oncogene-induced senescence. Senescence plays an important role in protecting the tumorous transformation of a living cell by oncogene activation via up-regulation of p53, p21 and p19 [23,24]. We examined p53 levels and found a striking reduction of p53 at steady state (Figure 6B). Consequently, p53 transcriptionally down-regulated its downstream target $\mathrm{p} 21^{\mathrm{Cip} 1}$ and indeed we found less $\mathrm{p} 21^{\mathrm{Cip} 1}$ in $\mathrm{eIF-5A2}$ MEFs. p19 ${ }^{\mathrm{Arf}}$ interferes with p53 negative regulator $\mathrm{Mdm} 2$ and therefore induces and stabilizes p53 [25,26]. It has been reported that the absent of p19 can enhance Mdm2's ability to down-regulate p53 during stress. We found a reduction of p19 at the protein level in eIF-5A2 MEFs, suggesting that $e I F-5 A 2$ activation serves as oncogenic stressor and thus repressing p19 (Figure 6B). Subsequently, CDK4, the down stream cell cycle molecule of p19 inhibitor, was upregulated in transgenic MEFs (Figure 6B), suggesting that in vitro overexperssion of eIF-5A2 contributes to oncogenic transformation, but does not drive the onset of cellular senescence.

\section{Activation of elF-5A2 caused partial transformation in MEFs}

Due to the massive overexpression of eIF-5A2 and reduced levels of p53 in transgenic MEFs, we wondered whether these together would favor cell proliferation. Therefore, firstly transgenic and wild-type MEFs were continuously passaged and monitored the replicative capability. Both MEFs proliferated normally until passage 4 and the proliferation declined during later passages (passage 5 and 6) (Figure 6C). Next we used an
$\mathrm{XTT}$ assay to compare cell growth rates between eIF$5 A 2$ transgenic MEFs and wild-type MEFs. The cell proliferating capability of transgenic MEFs was similar to that of wild-type MEFs when fed with $10 \%$ serum (Figure 6D). However, eIF-5A2 transgenic MEFs grew significantly faster than wild-type MEFs in low-serum (1\%) conditions $(p<0.05$, Figure 6E).

Telomerase has been detected in human cancer cells and is more active in transformed cells than in normal cells. This provides a selective growth advantage in transformed cells. Given the facts that the activation of eIF-5A2 and down-regulation of tumor suppressors p19 and p53, we hypothesized that eIF-5A2 MEFs could have increased telomerase activity. We used a telomere repeat amplification assay (TRAP) to compare the telomerase activities between transgenic and wild-type MEFs. Interestingly, 6 out of $8(75 \%)$ tested transgenic MEFs displayed increased telomerase activity compared to 7 wild-type controls (Figure 6F). Taken together, in in vitro culture we obtained typical, at least partial, phenotypes similar to transformed cells in transgenic MEFs overexpressing eIF$5 \mathrm{~A} 2$, which is consistent with our previous results $[7,8]$.

\section{Chromosomal instability in elF-5A2 transgenic mice}

Considering the role of p53 in maintenance of genetic stability and its down-regulation in eIF-5A2 transgenic mice, the correlation of eIF-5A2 expression and chromosomal instability was investigated. Metaphases from MEFs and adult mouse bone marrow were cytogenetically characterized and compared between eIF-5A2 transgenic mice and their wild-type littermates. Karyotyping analysis showed that the frequency of aneuploidy cells was significantly higher in transgenic MEFs $(18.5 \%, \mathrm{n}=$ 200) than that in wild-type MEFs (4.5\%, $\mathrm{n}=200, p<$ $0.05)$. In adult mouse bone marrow cells, the frequency of aneuploidy cells was significantly higher in transgenic mice $(11.5 \%, \mathrm{n}=200)$ than that in wild-type mice $(3 \%, \mathrm{n}$ $=200, p<0.05)$. Spectral karyotyping (SKY) analysis also found an increased number of aneuploidy cells in adult transgenic mice (16/100 metaphases) compared to wildtype controls (6/100 metaphases) (Figure 7A).

The dividing MEF nuclei from both eIF-5A2 transgenic mice and their wild-type littermates were also compared. We found a significant increase in misaligned chromosomal material in transgenic MEFs $(3.1 \%, \mathrm{n}=1,000)$ compared to wild-type controls $(1.2 \%, \mathrm{n}=1,000, p<0.05)$ (Figure 7B). Lagging chromosomal material, in the form of an anaphase bridge (Figure 7B), was detected in $4.1 \%$ $(\mathrm{n}=1,000)$ of transgenic MEFs, but not in wild-type MEFs. In addition, the detection of micronuclei after mitosis was significantly higher in eIF-5A2 transgenic mice $(5.6 \%, \mathrm{n}=1,000)$ than in the wild-type controls $(2.2 \%, \mathrm{n}=1,000 ; p<0.001$, Figure $7 \mathrm{C}$ and $7 \mathrm{D})$. 


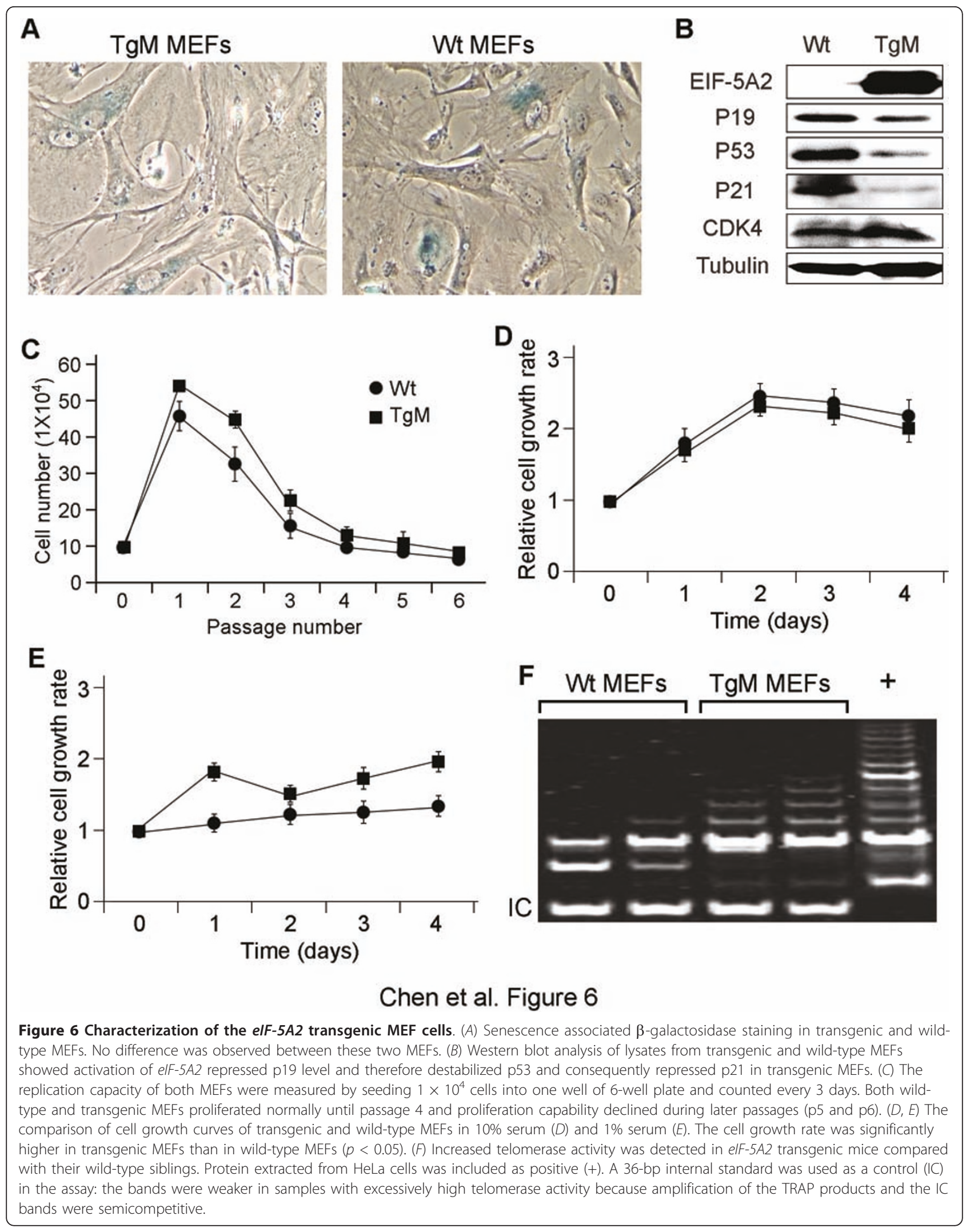




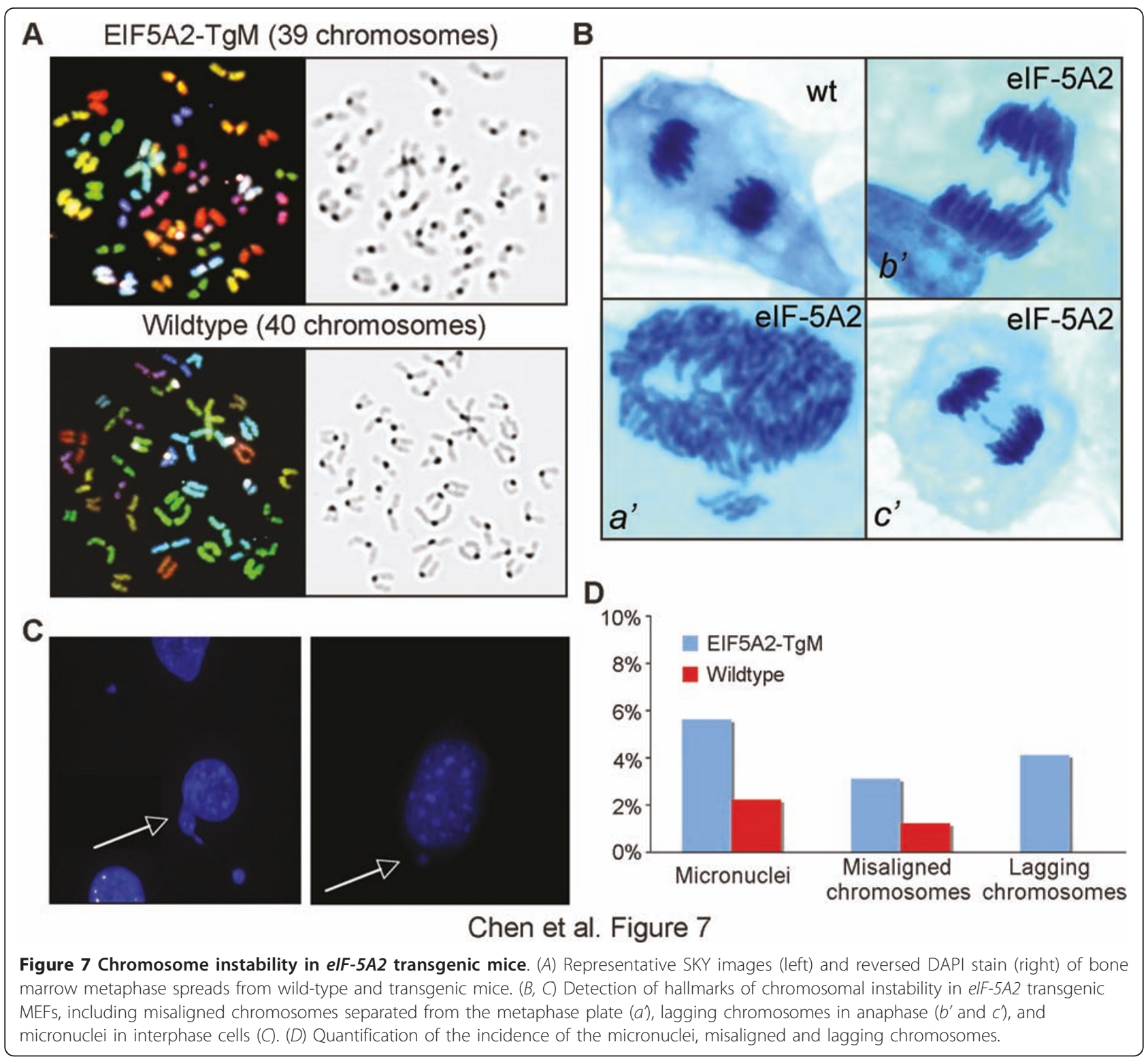

\section{Discussion}

Our studies have identified $e I F-5 A 2$ as a single genetic change involved in accelerating the aging process in mice. Overexpression of $e I F-5 A 2$ results in aging phenotypes in multiple organs in vivo, but is insufficient for triggering cellular senescence. We provide here the mammalian genetic evidence for the notion that organismal aging is not always linked to cellular senescence.

A key finding in our study is that activation of oncogene eIF-5A2 represses p19 levels and impairs its stabilization of p53. Consequently, p53 transcriptionally down-regulates $\mathrm{p} 21^{\mathrm{Cip} 1}$ and therefore increases CDK4 in response to p19 inhibition. However, these eIF-5A2 mediated pathway alterations function very differentially in in vivo and in vitro contexts. In vivo, down-regulated p19, p53 and p21 and up-regulated CDK4 promote cell proliferation and increase telomere activity, which belong to oncogenic transformation category. Consequently, we observed partial transformation of eIF-5A2 MEFs, but not oncogene activation induced cellular senescence. In the mouse, the activation of eIF-5A2 and subsequent alteration of the p19-p53-p21 pathway, leads to accelerated aging phenotypes. This confirms again the fate of oncogene activation is context dependent. The activation of eIF-5A2 alone in an in vitro system shows a mild transformation capability and is insufficient to trigger the senescence response. While in the intact mice context, the stress of eIF-5A2 activation causes whole body responses and navigates to accelerated aging to suppress tumorigenesis in vivo. As a result, 
there is no spontaneous tumor formation in eIF-5A2 mice, and they are not cancer-prone, and they do not respond to cancer reagents.

Notably, the p19 mediated impairment of p53, allowing the accumulation of numerical genomic instability, was revealed in both MEFs and mice. Human and mouse models of accelerated aging frequently involve alterations in genome maintenance mechanisms [21]. p53 plays a critical role in cell cycle regulation and the maintenance of genetic stability, which is associated with its function in DNA damage reparation. Genomic instability has been implicated as a major causal factor in early onset of the aging phenotype, which was observed in $\mathrm{mtr}-/$ - mice and Ku80 null mice $[27,28]$. Therefore, we hypothesized that the molecular mechanism of eIF-5A2 in aging is associated with p53-dependent chromosomal instability. The data we collected from both in vivo and in vitro studies showed a variety of chromosomal instability, including higher incidences of unaligned and/or misaligned chromosomal materials, anaphase bridges, and micronuclei. They contribute to differential fates of either aging or transformation depending on the different contexts, confirming the curial role of genomic instability in both tumorigenesis and aging.

eIF-5A2 was originally identified as a proto-oncogene whose overexpression leads to cancerous transformation of hepatocellular carcinoma cell lines, and contributes to cancer progression and metastasis. However, no spontaneous tumors were detected in transgenic mice overexpressing eIF-5A2. One possible explanation could be the life span of the eIF-5A2 transgenic mouse is too short to allow for the accumulation of extra genetic changes, and defeat aging phenotypes, to form a detectable tumor. In conclusion, we found that activation of eIF-5A2 causes p53-mediated genomic instability and accelerates the aging phenotype in multiple tissues in mice. This finding provides more insight into the mechanism of accelerated aging caused by oncogene activation in vivo.

Our study also revealed that cellular senescence is not required for the organismal aging process. Cellular senescence was considered as a potential counterpart of organismal aging under certain circumstances [29-31]. Senescent cells have typical physiological changes, including large and flat morphology, higher acidic $\beta$ galactosidase enzymatic activity and profound growth defects. The growth arrest of cellular senescence is actually a tumor suppressor mechanism to resist tumorigenesis via shortening the longevity of cells and preventing cell proliferation. In addition, senescences often downregulate extracellular matrix production and upregulate inflammatory cytokines to modulate the microenviroment or immune response [13]. Although we observed striking aging phenotypes in $e I F-5 A 2$ mice, there is no evidence that the aging effect of $e I F-5 A 2$ is associated with cellular senescence. Our model demonstrates that cellular senescence is not required for the initiation of eIF-5A2 mediated organismal aging. In another words, organismal aging is not necessarily the consequence of the accumulated senescent cells. This type of separation of cellular senescence and organism aging can be cell type and context dependent.

\section{Conclusions}

In the past several decades, age has been regarded as the largest risk factor tightly linked to the initiation of cancer. Cellular senescence, a potential in vitro counterpart of organismal aging, contributes to the cytotoxicity of certain anticancer agents. Here, we reveal for the first time a role of putative oncogenic eIF-5A2 in accelerating the aging process by increasing chromosome instability, and cellular senescence is not required for the aging phenotypes in eIF-5A2 mice. Thus, we conclude that organismal aging is not necessarily the consequence of the accumulated senescent cells. This type of separation of cellular senescence and organism aging can be cell type and context dependent.

\section{Acknowledgements}

This work was support by Hong Kong Research Grant Council Grant (HKU7656/07M), Grants from the Major State Basic Research Program of China (2006CB910104) and from National Natural Science Foundation of China (No. 30772475).

\section{Author details}

'Department of Clinical Oncology, Faculty of Medicine, The University of Hong Kong, 21 Sassoon Road, Hong Kong, China. ${ }^{2}$ Department of Biochemistry, Faculty of Medicine, The University of Hong Kong, 21 Sassoon Road, Hong Kong, China. ${ }^{3}$ Department of Cell Biology, College of Life Sciences, Peking University, Beijing, China. ${ }^{4}$ Department of Anatomy, Faculty of Medicine, The University of Hong Kong, 21 Sassoon Road, Hong Kong.

\section{Authors' contributions}

$\mathrm{MH}, \mathrm{JD}$ and $\mathrm{HK}$ performed the majority of experiments. WD, LL and SY carried out the cytogenetic studies. SS participated in the generation of transgenic mice. SL, TZ and GX helped with bone X-ray imaging analysis. XY supervised this project and provided suggestions. MH, SS and XY drafted and revised the manuscript. All authors reviewed, critiqued and offered comments to the text and approved the final version of manuscript.

\section{Competing interests}

The authors declare that they have no competing interests.

Received: 31 May 2010 Accepted: 26 May 2011 Published: 26 May 2011

\section{References}

1. Sonoda G, Palazzo J, du Manoir S, Godwin AK, Feder M, Yakushiji M, Testa JR: Comparative genomic hybridization detects frequent overrepresentation of chromosomal material from $3 q 26,8 q 24$, and 20q13 in human ovarian carcinomas. Genes Chromosomes Cancer 1997, 20:320-8.

2. Tai AL, Yan WS, Fang Y, Xie D, Sham JS, Guan XY: Recurrent chromosomal imbalances in nonsmall cell lung carcinoma: the association between $1 \mathrm{q}$ amplification and tumor recurrence. Cancer 2004, 100:1918-27.

3. Pack SD, Karkera JD, Zhuang Z, Pak ED, Balan KV, Hwu P, Park WS, Pham T, Ault DO, Glaser M, Liotta L, Detera-Wadleigh SD, Wadleigh RG: Molecular cytogenetic fingerprinting of esophageal squamous cell carcinoma by 
comparative genomic hybridization reveals a consistent pattern of chromosomal alterations. Genes Chromosomes Cancer 1999, 25:160-8.

4. Sattler HP, Rohde V, Bonkhoff H, Zwergel T, Wullich B: Comparative genomic hybridization reveals DNA copy number gains to frequently occur in human prostate cancer. The Prostate 1999, 39:79-86.

5. Forozan F, Mahlamaki EH, Monni O, Chen Y, Veldman R, Jiang $Y$, Gooden GC, Ethier SP, Kallioniemi A, Kallioniemi OP: Comparative genomic hybridization analysis of 38 breast cancer cell lines: a basis for interpreting complementary DNA microarray data. Cancer Res 2000, 60:4519-25.

6. Fang $Y$, Guan $X$, Guo Y, Sham J, Deng M, Liang Q, Li H, Zhang H, Zhou H, Trent J: Analysis of genetic alterations in primary nasopharyngeal carcinoma by comparative genomic hybridization. Genes Chromosomes Cancer 2001, 30:254-60.

7. Guan XY, Sham JST, Tang TCM, Fang Y, Huo KK, Yang JM: Isolation of a Novel Candidate Oncogene within a Frequently Amplified Region at 3 q26 in Ovarian Cancer. Cancer Res 2001, 61:3806-9.

8. Guan XY, Fung JMW, Ma NF, Lau SH, Tai LS, Xie D, Zhang Y, Hu L, Wu QL, Fang Y, Sham JST: Oncogenic Role of elF-5A2 in the Development of Ovarian Cancer. Cancer Res 2004, 64:4197-200.

9. Clement PM, Henderson CA, Jenkins ZA, Smit-McBride Z, Wolff EC, Hershey JW, Park MH, Johansson HE: Identification and characterization of eukaryotic initiation factor 5A-2. European journal of biochemistry/FEBS 2003, 270:4254-63.

10. Marchet A, Mocellin S, Belluco C, Ambrosi A, DeMarchi F, Mammano E, Digito M, Leon A, D'Arrigo A, Lise M, Nitti D: Gene expression profile of primary gastric cancer: towards the prediction of lymph node status. Annals of surgical oncology 2007, 14:1058-64.

11. Zender L, Xue W, Zuber J, Semighini CP, Krasnitz A, Ma B, Zender P Kubicka S, Luk JM, Schirmacher P, McCombie WR, Wigler M, Hicks J, Hannon GJ, Powers S, Lowe SW: An oncogenomics based in vivo RNAi screen identifies tumor suppressors in liver cancer. Cell 2008, 135:852-64.

12. DePinho RA: The age of cancer. Nature 2000, 408:248-54.

13. Campisi J: Aging and cancer: the double-edged sword of replicative senescence. Journal of the American Geriatrics Society 1997, 45:482-8.

14. Geigl JB, Langer S, Barwisch S, Pfleghaar K, Lederer G, Speicher MR: Analysis of gene expression patterns and chromosomal changes associated with aging. Cancer Res 2004, 64:8550-7.

15. Busuttil RA, Dolle M, Campisi J, Vijga J: Genomic instability, aging, and cellular senescence. Annals of the New York Academy of Sciences 2004, 1019:245-55.

16. Hasty P, Campisi J, Hoeijmakers J, van Steeg H, Vijg J: Aging and genome maintenance: lessons from the mouse? Science 2003, 299:1355-9.

17. Campisi J, d'Adda di Fagagna F: Cellular senescence: when bad things happen to good cells. Nature reviews 2007, 8:729-40.

18. Trueman D, Jackson SW, Trueman B: An automated technique for double staining rat and rabbit fetal skeletal specimens to differentiate bone and cartilage. Biotech Histochem 1999, 74:98-104.

19. Deng W, Tsao SW, Guan XY, Lucas JN, Si HX, Leung CS, Mak P, Wang LD, Cheung AL: Distinct profiles of critically short telomeres are a key determinant of different chromosome aberrations in immortalized human cells: whole-genome evidence from multiple cell lines. Oncogene 2004, 23:9090-101.

20. Deng W, Tsao SW, Lucas JN, Leung CS, Cheung AL: A new method for improving metaphase chromosome spreading. Cytometry A 2003, 51:46-51.

21. Vijg J: Somatic mutations and aging: a re-evaluation. Mutat Res 2000, 447:117-35.

22. Cao L, Li W, Kim S, Brodie SG, Deng CX: Senescence, aging, and malignant transformation mediated by p53 in mice lacking the Brca full-length isoform. Genes Dev 2003, 17:201-13.

23. Ferbeyre G, Lowe SW: The price of tumour suppression? Nature 2002, 415:26-7.

24. Xue W, Zender L, Miething C, Dickins RA, Hernando E, Krizhanovsky V, Cordon-Cardo C, Lowe SW: Senescence and tumour clearance is triggered by p53 restoration in murine liver carcinomas. Nature 2007, 445:656-60.

25. Quelle DE, Ashmun RA, Hannon GJ, Rehberger PA, Trono D, Richter KH, Walker C, Beach D, Sherr CJ, Serrano M: Cloning and characterization of murine p16INK4a and p15INK4b genes. Oncogene 1995, 11:635-45.
26. Kamijo T, Zindy F, Roussel MF, Quelle DE, Downing JR, Ashmun RA, Grosveld G, Sherr CJ: Tumor suppression at the mouse INK4a locus mediated by the alternative reading frame product p19ARF. Cell 1997, 91:649-59.

27. Qi L, Strong MA, Karim BO, Huso DL, Greider CW: Telomere fusion to chromosome breaks reduces oncogenic translocations and tumour formation. Nat Cell Biol 2005, 7:706-11.

28. Vogel H, Lim DS, Karsenty G, Finegold M, Hasty P: Deletion of Ku86 causes early onset of senescence in mice. Proc Natl Acad Sci USA 1999, 96:10770-5.

29. Smith JR, Pereira-Smith OM: Replicative senescence: implications for in vivo aging and tumor suppression. Science 1996, 273:63-7.

30. Campisi J: Cellular senescence as a tumor-suppressor mechanism. Trends in cell biology 2001, 11:S27-31.

31. van Heemst D, Mooijaart SP, Beekman M, Schreuder J, de Craen AJ, Brandt BW, Slagboom PE, Westendorp RG: Variation in the human TP53 gene affects old age survival and cancer mortality. Experimental gerontology 2005, 40:11-5.

\section{Pre-publication history}

The pre-publication history for this paper can be accessed here: http://www.biomedcentral.com/1471-2407/11/199/prepub

\section{doi:10.1186/1471-2407-11-199}

Cite this article as: Chen et al.: Overexpression of elF-5A2 in mice causes accelerated organismal aging by increasing chromosome instability. BMC Cancer 2011 11:199.

\section{Submit your next manuscript to BioMed Central and take full advantage of:}

- Convenient online submission

- Thorough peer review

- No space constraints or color figure charges

- Immediate publication on acceptance

- Inclusion in PubMed, CAS, Scopus and Google Scholar

- Research which is freely available for redistribution

Submit your manuscript at www.biomedcentral.com/submit
Ciomed Central 